\title{
BMJ Open Impact of chronic obstructive pulmonary disease on patients with aortic aneurysms: a nationwide retrospective cohort study in Taiwan
}

\author{
Kuang-Ming Liao, ${ }^{1}$ Chung-Yu Chen ${ }^{2,3}$
}

To cite: Liao K-M, Chen C-Y. Impact of chronic obstructive pulmonary disease on patients with aortic aneurysms: a nationwide retrospective cohort study in Taiwan. BMJ Open 2017;7:e015806. doi:10.1136/ bmjopen-2016-015806

- Prepublication history for this paper is available online. To view these files please visit the journal online (http://dx.doi. org/10.1136/bmjopen-2016015806).

Received 2 January 2017 Revised 23 June 2017 Accepted 19 July 2017

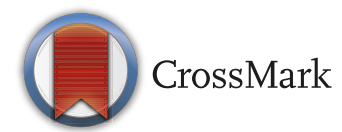

${ }^{1}$ Department of Internal Medicine, Chi-Mei Medical Center, Chiali, Taiwan ${ }^{2}$ Department of Pharmacy, Kaohsiung Medical University Hospital, Kaohsiung, Taiwan ${ }^{3}$ School of Pharmacy, Master Program in Clinical Pharmacy, Kaohsiung Medical University, Kaohsiung, Taiwan

Correspondence to Chung-Yu Chen jk2975525@hotmail.com

\section{ABSTRACT}

Objectives Aortic aneurysm (AA) is a leading cause of death worldwide. Chronic obstructive pulmonary disease (COPD) is a risk factor for $A A$, and the prognoses of COPD patients with $A A$ who underwent/did not undergo an operation warrant investigation.

Design A nationwide retrospective cohort study. Setting We included patients with AA older than 18 years who received their first AA diagnosis between 2005 and 2011 in Taiwan.

Participants This study enrolled 3263 COPD patients with AA before propensity score matching and 2127 COPD patients with AA after propensity score matching. Outcome measures The main outcomes were all-cause mortality and rehospitalisation for AA or operation. The outcomes of COPD patients with AA and COPD patients without AA during an 8-year follow-up period were examined using Cox proportional hazards models. Results In the AA population, patients with COPD showed higher rates of mortality and rehospitalisation than patients without COPD with adjusted HRs of $1.12(95 \% \mathrm{Cl} 1.03$ to 1.22) and 1.11 (95\% Cl 1.01 to 1.23 ), respectively, after propensity score matching. Analysis of the patients who underwent an operation revealed that the rates of mortality of COPD and non-COPD patients were not significantly different. In contrast, among the patients who did not receive an operation, patients with COPD showed a higher mortality rate than patients without COPD with an adjusted HR of 1.11 (95\% $\mathrm{Cl} 1.0$ to 1.22$)$.

Conclusions The outcomes of COPD patients with AA undergoing an operation were improved, but the mortality rate of non-COPD patients with $A A$ remained high. An effective treatment to reduce mortality in this group warrants further investigation.

\section{INTRODUCTION}

Chronic obstructive pulmonary disease (COPD) is an obstructive airway disease that is not fully reversible by medication. In fact, COPD is a systemic disease that shares smoking as a common risk factor for inducing aortic aneurysms (AAs); additionally, COPD has been shown to be an independent risk factor for abdominal aortic aneurysm (AAA). ${ }^{1}$ AAs include those of the abdominal aorta (AAA),

\section{Strengths and limitations of this study}

- This study is a nationwide cohort study that enrolled most aortic aneurysm (AA) patients with long-term follow-up over a period of 8 years.

- We used propensity score matching to reduce selection bias in patients with AA by balancing covariates between patients with and without chronic obstructive pulmonary disease (COPD).

- This is a comprehensive national study with a large population that intended to be representative of $A A$ patients with and without COPD in a clinical setting.

- The study relies on diagnosed COPD; however, according to a previous study, a large proportion of cases might be missed

- Some details regarding COPD and AA, such as lung function, aneurysm size and the presence of a dissection/rupture, might impact mortality; however, these were not assessed in our analyses.

thoracic aorta (TAA) and thoracoabdominal aorta (TAAA) and are associated with high mortality, particularly following rupture..$^{2-4}$

Previous studies have focused on the prognosis of patients with COPD undergoing operation for $\mathrm{AAA}^{5}$ or have compared the outcomes of COPD patients with AAA undergoing open or endovascular repair. ${ }^{6}$ However, these studies only considered the effect of COPD in AAA patients who underwent an operation, and some studies were performed more than 10 years ago. In addition, patients with AA may receive either surgical or medical treatment, but few studies have addressed the effect of COPD on patients with AA who receive medication. A previous study among AAA patients found that the risk of death was significantly greater in the presence of COPD. ${ }^{7}$ Another study showed that severe COPD, for which patients were dependent on home oxygen, was not a contraindication to AAA repair and that the mortality of these patients did not increase. ${ }^{8}$ Unfortunately, generalisation of these observational studies 
was limited by their small sample sizes, short follow-up periods, lack of medical records and conflicting results. Moreover, improvements in intraoperative and postoperative care for vascular operation have resulted in significant reductions in morbidity and mortality that might affect the outcomes of AA in patients with COPD. These factors justify further investigation.

To investigate the outcomes (all-cause mortality, rehospitalisation for $\mathrm{AA}$ and operation) of $\mathrm{AA}$ patients (including AAA, TAAA and AAA) with COPD, we used data from the Taiwanese National Health Insurance Research Database (NHIRD) for patients receiving either surgical or medical treatment. The aim of our study was to analyse the association between aortic aneurysm and COPD regardless of the risk factors of aortic aneurysm. The main outcomes were all-cause mortality and rehospitalisation for AA or operation. The hypothesis was that COPD has a large influence on the outcome of AAs.

\section{MATERIALS AND METHODS Study design}

This was a population-based, observational study using information from the NHIRD in Taiwan. Data regarding hospital visits, emergency care and prescriptions were provided by the National Health Insurance Research Institutes. The diagnosis of AA in the NHIRD has previously been described. ${ }^{9}$ This study was approved by the Institutional Review Board of Kaohsiung Medical University Hospital (KMUH-IRB-EXEMPT-20130199).

\section{Participants}

We included patients from the NHIRD aged $\geq 18$ years who presented with a first-time diagnosis of AA (International Statistical Classification of Diseases, Ninth Revision (ICD-9) 441.1-441.9) between 2005 and 2011. The date of the first ambulatory visit or inpatient hospitalisation at which AA was diagnosed was designated the index date. We excluded patients who died at first presentation and those who had fewer than 30 days of follow-up after discharge.

Participants included as cases were required to have a diagnosis of COPD (ICD-9: 490-492, 496) before the index date and treated using COPD medications including the following: long-acting inhaled anticholinergics, longacting inhaled $\beta 2$-adrenergic receptor agonists, inhaled corticosteroids, short-acting anticholinergics, short-acting $\beta 2$-agonists or xanthines. Patients were excluded if COPD was diagnosed or medications for COPD were prescribed after the index date.

The control group was selected from the group of patients with AA who had no comorbid diagnosis of COPD. To decrease selection bias due to baseline differences, we also conducted propensity score matching (PSM) at a 1:1 ratio by age group, demographic characteristics, comorbidities and comedications. The patients in this study were followed until readmission or operation for AA, death, withdrawal from the national health insurance or 31 December 2012, whichever was soonest.

\section{Variables}

All patients were divided into the TAA (ICD-9 codes 441.1-441.2), AAA (ICD-9 codes 441.3-441.4) and TAAA (ICD-9 codes 441.6-441.7) groups according to the diagnosis site at the index date. We also identified whether patients underwent operation and, if so, whether the operation involved open or endovascular repair.

Patient comorbidities were identified by the diagnostic code as inpatient or outpatient diagnoses within 180 days of the index date. The following comorbidities were included in the assessment: hypertension, diabetes, dyslipidaemia, congestive heart failure (CHF), atrial fibrillation, valvular heart disease (VHD), peripheral artery disease $(\mathrm{PAD})$, coronary artery disease $(\mathrm{CAD})$, stroke (ischaemic and haemorrhagic), malignancy, chronic kidney disease (CKD), thyroid disease, liver disease, gout, peptic ulcer disease and sleep apnoea. The drugs prescribed within 180 days of the index date were also identified. The following medications were also included as variables: ACE inhibitors (ACEIs), angiotensin receptor blockers (ARBs), beta-blockers (BBs), calcium channel blockers (CCBs), diuretics, alpha-blockers, statins, fibrates, antiplatelet drugs and oral antidiabetic agents (OADs).

We also described the demographic characteristics of patients with AA by the presence or absence of a COPD diagnosis. Potential confounders included gender, age group ( $<50,50-59,60-69,70-79$ and over 80 years), urbanisation (urban or rural), income group and smoking status. The income groups were defined as low $(<\mathrm{NT} \$ 24000)$, middle $(\mathrm{NT} \$ 24000-\mathrm{NT} \$ 42000)$ or high $(>\mathrm{NT} \$ 42000)$ according to the individual monthly gross income during a 1-month period before the index date.

Because no information was available regarding smoking status, we used the mean percentage of city/county smoking rates available from the National Health Interview Survey (2005-2011). All subjects in this survey were randomly sampled and selected from different cities and counties to be interviewed by trained interviewers. ${ }^{10}$ Although this smoking rate is not a direct measure of the rate for patients in this database, it can be considered to reflect the likely rates of smoking and passive smoking (stratified by age, gender and living area) if we assume a normally distributed cohort that reflects the broader population.

\section{Outcome}

The main outcomes of interest were all-cause mortality and rehospitalisation for AA or operation. Moreover, all-cause mortality was confirmed by withdrawal from National Health Insurance (NHI) within 1 month of a major medical event. ${ }^{11}$ We considered rehospitalisation to be the time when patients were admitted with AA-related events (ICD-9 codes 441.1-441.9) as the principal or secondary diagnosis after discharge from the index event. Operation was stratified by whether patients underwent an operation at the time of the index event. Then, the patients who underwent an operation for AA at the index event and were rehospitalised for operation more than 30 days after discharge were classified as undergoing a repeat 
operation. Patients who did not undergo operation at the index event and who were rehospitalised more than 30 days after discharge were classified into the operation group. Furthermore, patients who received an operation less than 30 days after discharge at the index event were classified into the operation group at the index event.

\section{Statistics}

All data are expressed as frequencies (percentages). Categorical and continuous variables were compared between the patients with and without COPD using $\chi^{2}$ tests and Student's t-tests, as appropriate. The outcomes of the patients with and without COPD during an 8-year follow-up period were examined by Cox proportional hazards models.

Simple and multiple analyses (including age group, comorbidities, comedications and demographic characteristics) were used to assess the risk of outcome by the presence or absence of COPD during follow-up. We used PSM for patients in the COPD and non-COPD groups. Because the outcomes might have been influenced by the presence of disease (diabetes mellitus, dyslipidaemia and hypertension) or medication (OAD, statins, fibrates and antihypertensives), we examined these in a multiple model. The difference in the cumulative probability of mortality between the patients with and without COPD was calculated using Kaplan-Meier estimates with the log-rank test; we also divided patients by operation type and site of diagnosis for these analyses.

To assess the robustness of the outcomes, a subgroup analysis was performed, and the subgroups were defined by age ( $\geq 70$ and $<70$ years), AA site (TAA, AAA and TAAA), high risk (hypertension, dyslipidaemia, diabetes mellitus, atrial fibrillation, stroke and CKD), concomitant drug use (ACEIs, BBs, CCBs, statins and antiplatelet agents) and type of operation. The analyses and calculations were performed using SAS V.9.4. Statistical significance was inferred at a two-sided $\mathrm{p}$ value $<0.05$.

\section{RESULTS}

\section{Descriptive data}

We identified 6117 patients with AA, and 3263 of these patients also had COPD. After PSM, 2127 patients with COPD were eligible for enrolment in the study. Figure 1 shows the study flow diagram for AA patients with and without COPD.

Table 1 summarises the characteristics of patients with AA. Before matching, one-third of the patients with AA were aged 70-80 years. A notable male preponderance was detected in the patients with and without COPD.

In both groups, AAA was the most common type of AA, followed by TAA and TAAA. Most patients without COPD (58\%) received open repair, whereas most patients with COPD (55\%) underwent endovascular repair. The most common comorbidity was hypertension, which was present in more than $96 \%$ of patients.
The two most common medications administered to the patients with and without COPD were CCBs and diuretics, respectively.

\section{Outcomes based on the operation and non-operation groups}

As shown in table 2, among the patients with AA who received an operation, there were no significant differences in all-cause mortality between the patients with and without COPD. Among the non-operation group, the all-cause mortality was higher in patients with COPD compared with patients without COPD both before and after PSM, with respective adjusted HRs of 1.233 (95\% CI 1.130 to 1.344 ) and 1.105 (95\% CI 1.004 to 1.216$)$. The reoperation rate for $\mathrm{AA}$ was higher in the patients without COPD than in the patients with COPD with an adjusted HR of 3.134 (95\% CI 1.394 to 7.043) after PSM.

\section{Outcome of AAs by the presence or absence of COPD}

As shown in table 2, an analysis of the total population revealed that patients with COPD presented a higher all-cause mortality than patients without COPD. The AA rehospitalisation rate of the patients with COPD was also higher than that of the patients without COPD, with respective adjusted HRs before and after PSM of 1.100 (95\% CI 1.004 to 1.206 ) and 1.114 (95\% CI 1.007 to 1.2327). Among the total population, no significance difference in the number of patients who underwent an operation was found between the patients with and without COPD.

\section{Other factors affecting outcomes}

Table 3 shows the HRs and 95\% CI for all-cause mortality and rehospitalisation before and after PSM.

As shown in table 3 , COPD and age $\geq 80$ years were associated with higher mortality rates after PSM with respective HRs of 1.118 (95\% CI 1.028 to 1.217) and 2.970 (95\% CI 1.533 to 5.755$)$. In addition, AAA and TAAA were associated with a higher risk of rehospitalisation than TAA after PSM, and AAA was also associated with a lower risk of mortality than TAAA.

Other comorbidities associated with a higher risk of mortality after PSM included diabetes, dyslipidaemia, CHF, atrial fibrillation, VHD, stroke, malignancy, CKD and peptic ulcer disease. Among the patients with multiple comorbidities, only CAD and CKD were associated with a significantly higher risk of rehospitalisation.

Medications that showed an apparent protective effect on mortality after PSM were ARBs, BBs and CCBs. Patients prescribed CCBs had a lower risk of rehospitalisation, and those prescribed $\alpha$ blockers presented a higher risk of rehospitalisation. After PSM, AA patients had a lower risk of mortality if they were receiving ARBs than if they were receiving ACEIs, with HRs of 0.846 (95\% CI 0.766 to 0.934 ) and 1.170 (95\% CI 1.058 to 1.293). However, there was no significant difference in the rehospitalisation rates between patients receiving ACEIs and those receiving ARBs after PSM. 
Aortic aneurysm first-time diagnosed patients from national health insurance research database within 2005-2011, $\mathrm{n}=11,939$

Exclude patients $<18 \mathrm{y} / \mathrm{o}, \mathrm{n}=106$

No ambulatory visit or inpatient hospitalisation with a first-time aortic aneurysm, $n=1,998$

Exclude COPD diagnosed after index day, $\mathrm{n}=1.256$

Exclude death with a first-time aortic aneurysm diagnosed at ambulatory visit or inpatient hospitalisation $\mathrm{n}=1.262$

Exclude patients surviving less than 30 days after first-time aortic aneurysm discharge, $\mathrm{n}=1,200$

An ambulatory visit or inpatient hospitalisation with a first-time aortic aneurysm from national health insurance research database within 20052011. $n=6.117$
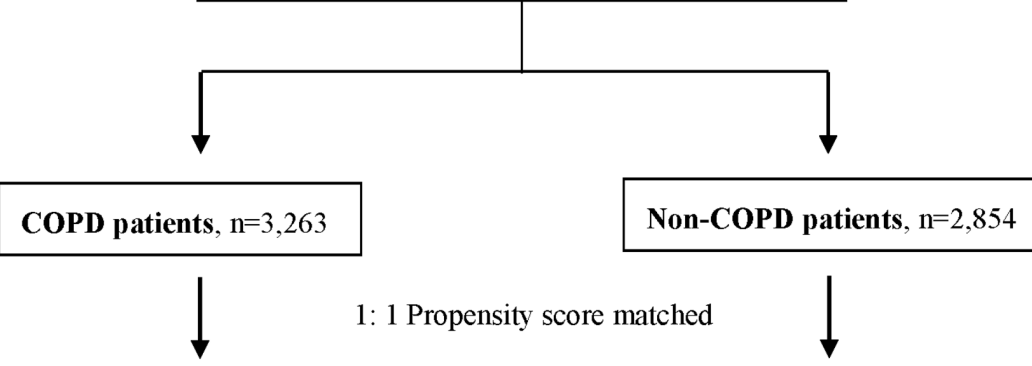

COPD patients, $n=2,127$

Non-COPD patients, $n=2,127$

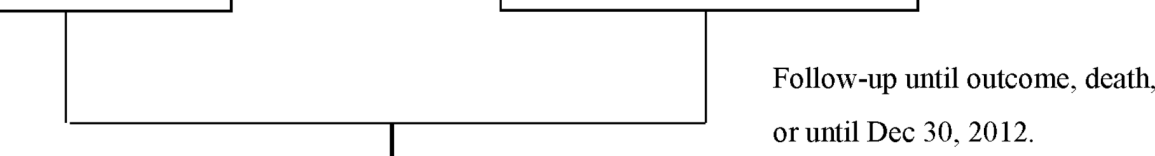

Outcome as all-cause of mortality, operation, and aortic aneurysm re-hospitalisation.

Figure 1 Study flow diagram. COPD, chronic obstructive pulmonary disease.

\section{Mortality analysis in patients with AA and COPD}

Table 4 shows the results of a subgroup analysis for mortality in patients with AA defined by the presence or absence of COPD. AA patients with COPD had higher mortality rates than those without COPD. Patients $\geq 70$ years of age (HR 1.117, 95\% CI 1.021 to 1.223 ) or with TAA (HR $1.26295 \%$ CI 1.075 to 1.482 ) also showed higher risks of mortality. The HR for mortality in the patients with AA, COPD and hypertension was also significant (HR 1.111, 95\% CI 1.019 to 1.211). Other comorbidities, including diabetes, dyslipidaemia, atrial fibrillation, stroke and CKD, were not associated with increased risks of mortality in AA patients with COPD. There was also no significant difference in the mortality rates between the operation procedures for patients with COPD and AA.

Figure 2 shows the cumulative risk of AA mortality in patients with and without COPD and is stratified by patients who underwent an operation and by the AA site. Analysis of the patients with TAAA who underwent an operation showed no significant difference in mortality between the patients with and without COPD ( $p=0.6516)$. However, for all other subgroups of patients with AA, there were significant differences in mortality based on the presence or absence of COPD. 
Table 1 Characteristics of patients with aortic aneurysms in Taiwan

\begin{tabular}{|c|c|c|c|c|c|c|}
\hline & $\begin{array}{l}\text { Non-COPD } \\
(n=2854)\end{array}$ & $\begin{array}{l}\text { COPD } \\
(n=3263)\end{array}$ & & $\begin{array}{l}\text { PS matching } \\
\text { Non-COPD }(n=2127)\end{array}$ & $\begin{array}{l}\text { PS matching COPD } \\
(\mathrm{n}=2127)\end{array}$ & \\
\hline Variable & No (\%) & No (\%) & $\mathbf{p}$ & No (\%) & & $\mathbf{p}$ \\
\hline Age group (year) & & & $<0.001$ & & & 0.436 \\
\hline$<50$ & $273(9.6)$ & $61(1.9)$ & & $64(3.0)$ & $61(2.9)$ & \\
\hline $50-60$ & $356(12.5)$ & $113(3.5)$ & & $134(6.4)$ & $112(5.3)$ & \\
\hline $60-70$ & $508(17.8)$ & $388(11.9)$ & & $327(15.4)$ & $354(16.6)$ & \\
\hline $70-80$ & $932(32.6)$ & $1183(36.2)$ & & $837(39.4)$ & $853(40.1)$ & \\
\hline$>80$ & $785(27.5)$ & $1515(46.5)$ & & $763(35.9)$ & $747(35.1)$ & \\
\hline Gender & & & $<0.001$ & & & 0.971 \\
\hline Female & $745(26.1)$ & $631(19.3)$ & & $509(23.9)$ & $508(23.9)$ & \\
\hline Male & 2109 (73.9) & $2632(80.7)$ & & $1618(76.1)$ & 1648 (76.1) & \\
\hline Urbanicity & & & $<0.001$ & & & 0.733 \\
\hline Urban & $724(25.4)$ & $1011(31.0)$ & & $592(27.8)$ & $602(28.3)$ & \\
\hline Rural & $2130(74.6)$ & 2252 (69.0) & & 1535 (72.2) & $1525(71.7)$ & \\
\hline Income (NT) & & & $<0.001$ & & & 0.831 \\
\hline Low $(<24000)$ & $2564(89.8)$ & $3136(96.1)$ & & 2002 (94.1) & 2011 (94.6) & \\
\hline Middle (24000-42 000) & 149 (5.2) & $74(2.3)$ & & $71(3.3)$ & $65(3.1)$ & \\
\hline High (>42 000) & $141(5.0)$ & $53(1.6)$ & & $54(2.5)$ & $51(2.4)$ & \\
\hline Aortic aneurysm site & & & $<0.001$ & & & 0.918 \\
\hline TAA & 965 (33.8) & 1018 (31.2) & & $664(31.2)$ & $662(31.1)$ & \\
\hline AAA & $1689(59.2)$ & $2089(64.0)$ & & $1355(63.7)$ & $1351(63.5)$ & \\
\hline TAAA & $200(7.0)$ & $156(4.8)$ & & $108(5.1)$ & $114(5.4)$ & \\
\hline Operation & $1181(41.4)$ & 1018 (31.2) & $<0.001$ & $823(38.7)$ & $753(35.4)$ & 0.026 \\
\hline Open repair & $689(58.3)$ & $455(44.7)$ & & $425(51.6)$ & 340 (45.2) & \\
\hline Endovascular repair & $492(41.7)$ & $563(55.3)$ & & $399(48.4)$ & $413(54.7)$ & \\
\hline Smoking rate & $26.3( \pm 13.4)$ & $28.6( \pm 12.3)$ & $<0.001$ & $27.0( \pm 13.1)$ & $27.0( \pm 13.1)$ & 0.921 \\
\hline \multicolumn{7}{|l|}{ Comorbidities } \\
\hline Diabetes & $414(14.5)$ & $450(13.8)$ & 0.423 & $310(14.6)$ & $307(14.4)$ & 0.896 \\
\hline Hypertension & 2766 (96.9) & 3138 (96.2) & 0.112 & 2055 (96.6) & 2051 (96.4) & 0.738 \\
\hline Dyslipidaemia & $944(33.1)$ & 1032 (31.6) & 0.227 & 727 (34.2) & $725(34.1)$ & 0.948 \\
\hline $\mathrm{CHF}$ & $561(19.7)$ & 1019 (31.2) & $<0.001$ & $488(22.9)$ & $482(22.7)$ & 0.826 \\
\hline Atrial fibrillation & $230(8.1)$ & $384(11.8)$ & $<0.001$ & $196(9.2)$ & $189(8.9)$ & 0.708 \\
\hline VHD & $594(20.8)$ & $556(17.0)$ & $<0.001$ & $377(17.7)$ & 368 (17.3) & 0.717 \\
\hline PAD & $152(5.3)$ & $222(6.8)$ & 0.016 & $128(6.0)$ & $139(6.5)$ & 0.487 \\
\hline CAD & 1135 (39.8) & $1685(51.6)$ & $<0.001$ & $965(45.4)$ & $974(45.8)$ & 0.782 \\
\hline Stroke & $736(25.8)$ & $1127(34.5)$ & $<0.001$ & $642(30.2)$ & $646(30.4)$ & 0.894 \\
\hline Malignance & $479(16.8)$ & $645(19.8)$ & 0.003 & 402 (18.9) & 407 (19.6) & 0.560 \\
\hline CKD & $453(15.9)$ & $597(18.7)$ & 0.012 & $374(17.6)$ & $378(17.8)$ & 0.872 \\
\hline Thyroid disease & $87(3.1)$ & $97(3.0)$ & 0.863 & $59(2.8)$ & $64(3.0)$ & 0.647 \\
\hline Liver disease & $343(12.0)$ & $441(13.5)$ & 0.081 & $267(12.6)$ & $254(11.9)$ & 0.543 \\
\hline Sleep apnoea & $317(11.1)$ & $533(16.3)$ & $<0.001$ & $271(12.7)$ & $268(12.6)$ & 0.890 \\
\hline Peptic ulcer disease & $257(9.0)$ & $361(11.1)$ & 0.007 & $215(10.1)$ & $218(10.3)$ & 0.879 \\
\hline Gout & $520(18.2)$ & $628(19.5)$ & 0.305 & $414(19.5)$ & $411(19.3)$ & 0.974 \\
\hline \multicolumn{7}{|l|}{ Prescribed drugs } \\
\hline ACEI & $691(24.2)$ & 857 (26.3) & 0.066 & $521(24.5)$ & $507(23.8)$ & 0.616 \\
\hline
\end{tabular}


Table 1 Continued

\begin{tabular}{|c|c|c|c|c|c|c|}
\hline & $\begin{array}{l}\text { Non-COPD } \\
(\mathrm{n}=2854)\end{array}$ & $\begin{array}{l}\text { COPD } \\
(n=3263)\end{array}$ & & $\begin{array}{l}\text { PS matching } \\
\text { Non-COPD }(n=2127)\end{array}$ & $\begin{array}{l}\text { PS matching COPD } \\
(\mathrm{n}=2127)\end{array}$ & \\
\hline Variable & No (\%) & No (\%) & $p$ & No (\%) & & $p$ \\
\hline ARB & 948 (33.2) & 1058 (32.4) & 0.510 & 695 (32.7) & 679 (31.9) & 0.600 \\
\hline BB & 1737 (60.9) & $1583(48.5)$ & $<0.001$ & $1174(55.2)$ & 1178 (55.4) & 0.902 \\
\hline ССВ & 2187 (76.6) & $2419(74.13)$ & 0.024 & 1607 (75.4) & 1616 (76.7) & 0.747 \\
\hline Diuretics & $1874(65.7)$ & 2158 (66.1) & 0.697 & $1371(64.5)$ & $1388(65.3)$ & 0.585 \\
\hline Alpha-blocker & 457 (16.0) & 541 (16.6) & 0.549 & 344 (16.2) & 346 (16.2) & 0.934 \\
\hline Statin & $480(16.8)$ & 535 (16.4) & 0.658 & 373 (17.5) & $378(17.8)$ & 0.841 \\
\hline Fibrate drugs & $68(2.4)$ & $61(1.9)$ & 0.163 & $41(1.9)$ & $42(1.9)$ & 0.912 \\
\hline Antiplatelet drugs & 1349 (47.3) & $1742(53.4)$ & $<0.001$ & $1083(50.9)$ & $1084(50.9)$ & 0.976 \\
\hline OAD & $236(8.3)$ & 293 (8.9) & 0.324 & $192(9.0)$ & $186(8.7)$ & 0.747 \\
\hline
\end{tabular}

AAA, abdominal aortic aneurysm; ACEI, ACE inhibitor; ARB, angiotensin receptor blocker; BB, beta-blocker; CAD, coronary artery disease; $\mathrm{CCB}$, calcium channel blocker; $\mathrm{CHF}$, congestive heart failure; CKD, chronic kidney disease; COPD, chronic obstructive pulmonary disease; $\mathrm{OAD}$, oral antidiabetic agent; PAD, peripheral artery disease; PS, propensity score; TAA, thoracic aortic aneurysm; TAAA, thoracoabdominal aneurysm; VHD, valvular heart disease.

\section{DISCUSSION}

This nationwide population-based study showed that AA patients with COPD had a higher risk of all-cause death and rehospitalisation than those without COPD. Analysis of the patients with AA who underwent an operation showed that those with COPD had the greatest risk of reoperation for AAs. However, no differences in mortality in AA patients with COPD were found among the different procedures for AA.

\section{Prognosis of AA patients with COPD}

A previous study showed that the increased prevalence of COPD in patients with AAA was independent of smoking. ${ }^{1}$ Other studies have found that COPD is associated with a high prevalence of AAA with rates ranging from $7.7 \%$ to $9.9 \%$ and that the prevalence is increased in severe emphysema and in cases with a decreased forced expiratory volume/vital capacity ratio. ${ }^{12} 13$ The aneurysm rupture rates have also been shown to correlate with COPD risk factors, initial aneurysm size and diastolic hypertension, ${ }^{14}$ but surgeons are hesitant to repair AAAs electively because of the associated high morbidity and mortality. ${ }^{15}$ Through a database review of 1053 patients undergoing surgery for intact or ruptured AAAs in a hospital between 1997 and 1998, Axelrod et a $\bar{l}$ showed that mortality was not higher in patients with COPD compared with patients without COPD. Notably, these researchers also showed that patients with COPD required longer hospital stays, longer intensive care unit stays and more days of ventilation. However, their study was performed more than 15 years ago and only focused on patients with AAA who underwent operations. Axelrod et a $\tilde{e}$ did not find an increased risk in patients undergoing surgery, which might be due to adequate patient selection in an era of open repair.

Our study included patients with a first-time AA diagnosis between 2005 and 2011 and who were followed to
31 December 2012. The final cohort of patients with AA comprised 2854 controls without COPD and 3263 cases with COPD, and these were further divided into those who did and did not undergo operation. Our study indicated that AA patients with COPD had a higher rate of rehospitalisation and higher mortality compared with patients without COPD. In patients undergoing operation for AAs, we found that the mortality and rehospitalisation rates were not significantly different between those with and without COPD, although the reoperation rate was higher in those with COPD. Among those not undergoing operation for AA, the all-cause mortality rate was also higher among patients with COPD than those without COPD.

\section{Surgical procedure and mortality in patients with COPD and AA}

Compton et $a l^{16}$ retrospectively reviewed 44 patients with oxygen-dependent COPD undergoing AAA repair; of these, 24 underwent endovascular aneurysm repair and 20 underwent open procedures. These researchers showed that the type of repair, comorbidities and lung function test results did not significantly affect survival. Many other studies have also shown that endovascular AAA repair offers long-term survival similar to open AAA repair in patients with COPD ${ }^{17-19}$ Qureshi $e t a t^{20}$ performed a prospective study of high-risk patients undergoing endovascular repair of AAAs or TAAAs between 1998 and 2009 and showed that mortality was no different between patients with and without COPD when endovascular techniques were used. In our study comparing all types of AA in patients with and without COPD, we found no significant difference in mortality rates for either procedure between the two groups.

\section{Patient characteristics}

In our study, patients with COPD had higher rates of AA than those without COPD. Most patients with AA were 


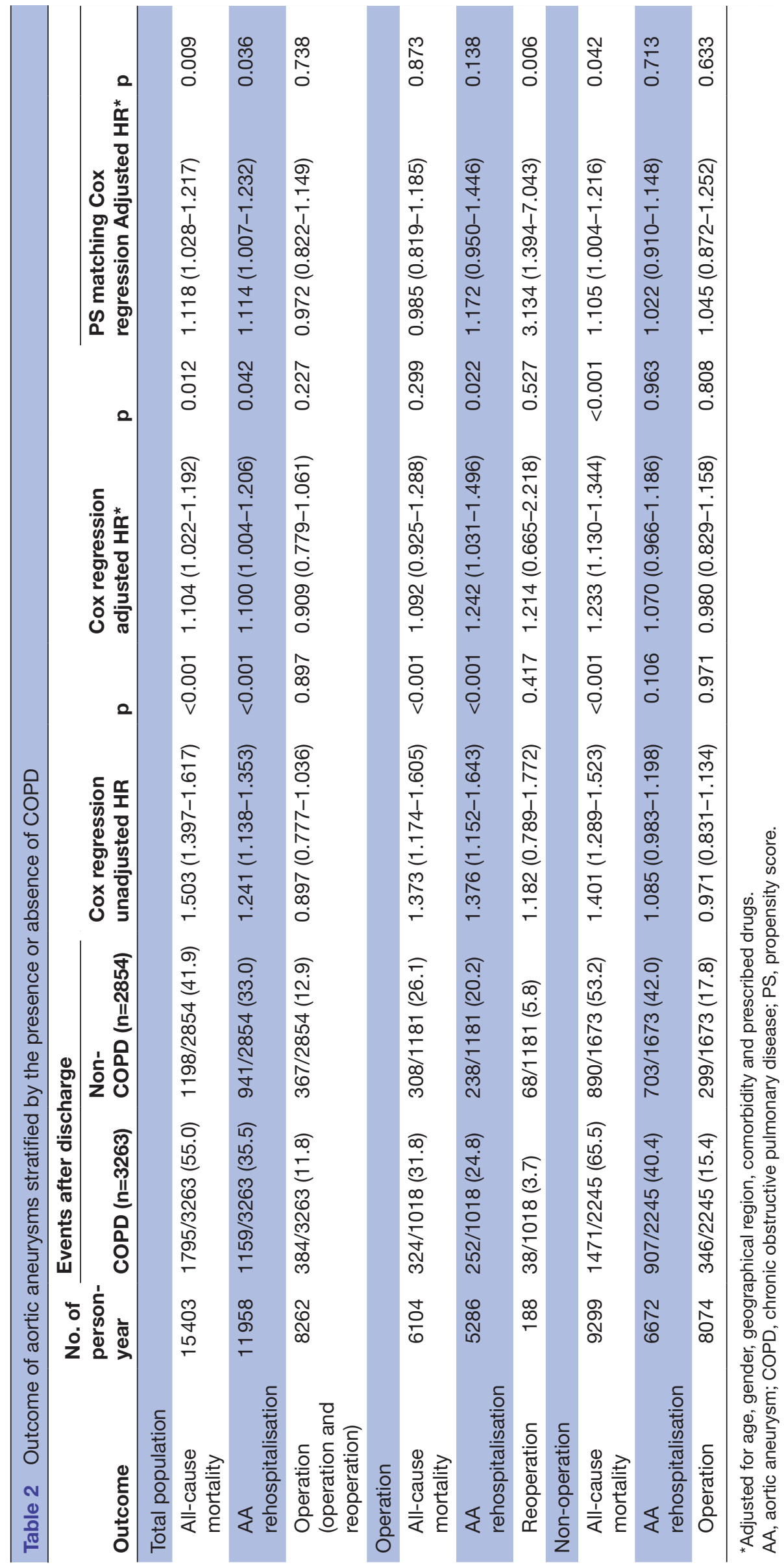




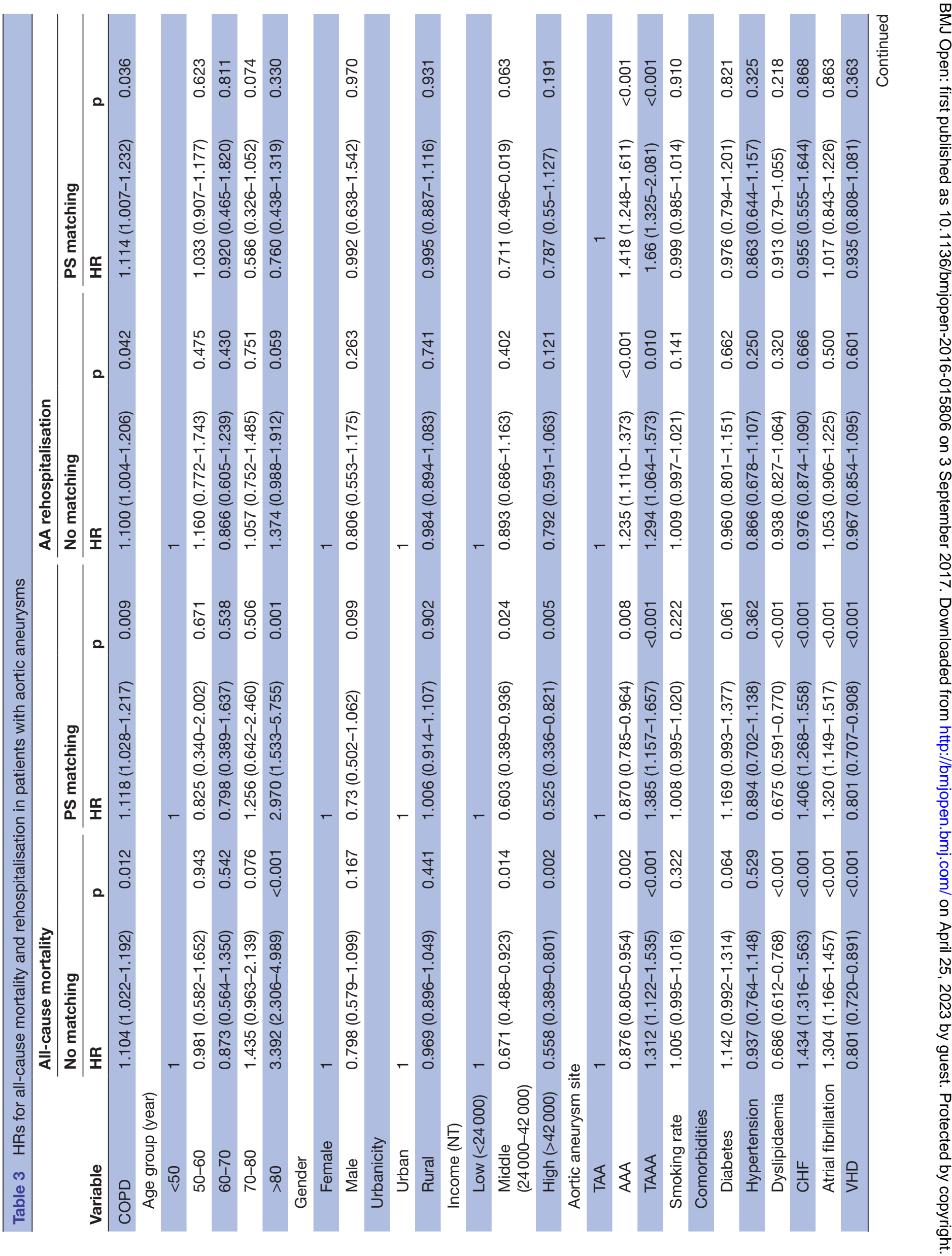




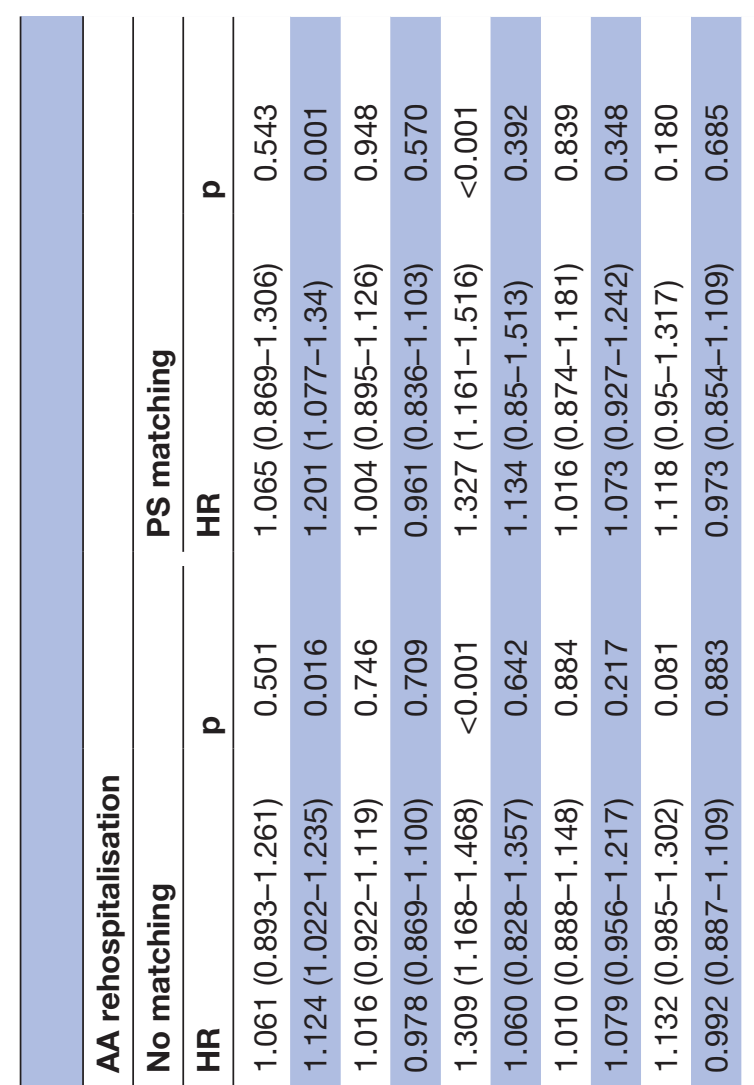

穴

ก̃

สิ

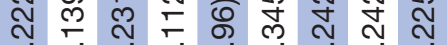

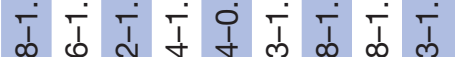

赑

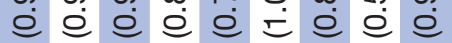

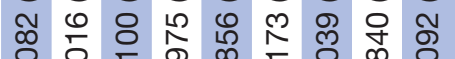

家 담

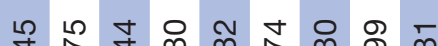

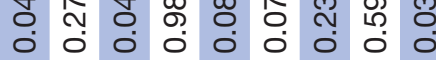

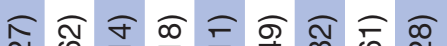

N

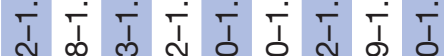

ชิ

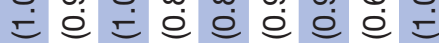

ภ เก

욷 눔 둠

孚응

$\Omega$

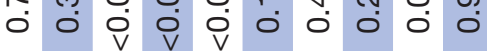

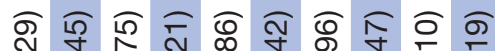

ก० ব

2)

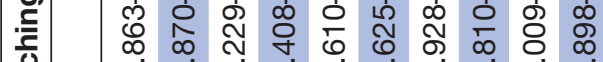

过

ह

๗

స్ ஸి

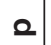

菅

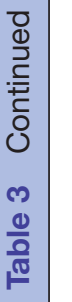

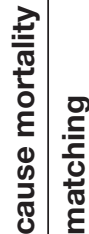

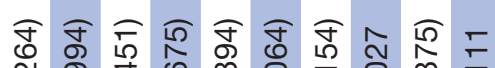
† ป i⿱

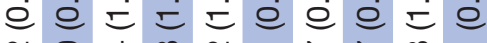

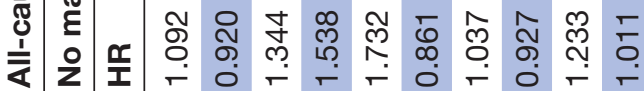

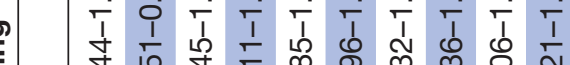

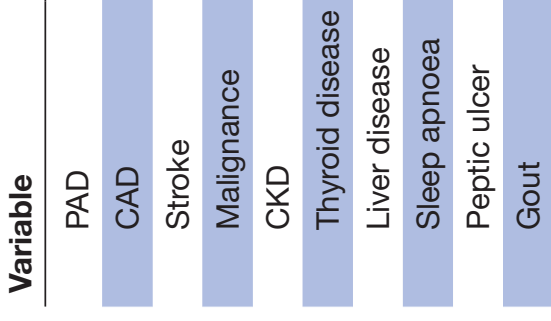

ชิ

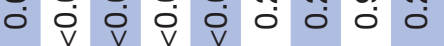

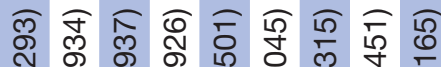

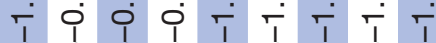

赵

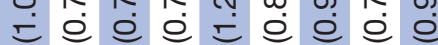

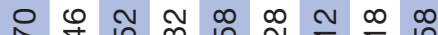

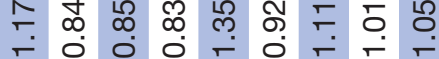

ธ

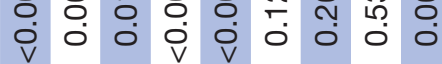

ติ

ヘָ

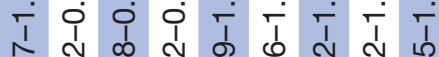

ก

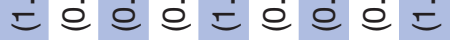

下

品

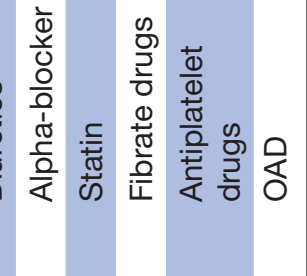

กิ

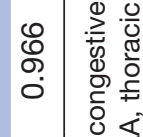

ลิ

क

गิ

$\infty$ ब

늘

S U

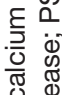

m.

ภ 00

○

ळ $\widetilde{\square}$

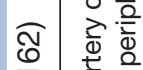

ᄃ.

กิ के व

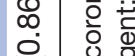

응

.

응

人

०

ลิ

ले 응

厂

$\infty$

远

色 क

ᄃ.

可

L

家

产 塞

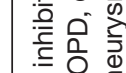

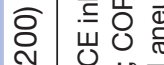

† बٓ

।

ชิ

है

8 은

ब.

은

बे

है

융

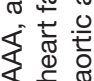


Table 4 Subgroup analysis for all-cause mortality in patients with aortic aneurysms and COPD

\begin{tabular}{|c|c|c|c|c|}
\hline \multirow[b]{3}{*}{ Variable } & \multicolumn{4}{|l|}{ COPD } \\
\hline & \multicolumn{2}{|l|}{ No matching } & \multicolumn{2}{|l|}{ PS matching } \\
\hline & HR & $p$ & HR & $\mathbf{p}$ \\
\hline COPD & $1.104(1.022-1.192)$ & 0.012 & $1.118(1.028-1.217)$ & 0.009 \\
\hline \multicolumn{5}{|l|}{ Age group (year) } \\
\hline$<70$ & $1.161(0.933-1.444)$ & 0.181 & $1.184(0.926-1.514)$ & 0.179 \\
\hline$\geq 70$ & $1.103(1.016-1.198)$ & 0.020 & $1.117(1.021-1.223)$ & 0.016 \\
\hline \multicolumn{5}{|l|}{ Aortic aneurysm site } \\
\hline TAA & $1.205(1.042-1.392)$ & 0.012 & $1.262(1.075-1.482)$ & 0.004 \\
\hline AAA & $1.048(0.952-1.154)$ & 0.340 & 1.055 (0.949-1.173) & 0.318 \\
\hline TAAA & $1.074(0.775-1.488)$ & 0.670 & $0.986(0.687-1.414)$ & 0.939 \\
\hline \multicolumn{5}{|l|}{ Comorbidities } \\
\hline Diabetes (yes) & $0.911(0.711-1.166)$ & 0.458 & $0.826(0.624-1.093)$ & 0.181 \\
\hline Diabetes (no) & $1.137(1.048-1.234)$ & 0.002 & $1.164(1.064-1.272)$ & $<0.001$ \\
\hline Hypertension (yes) & $1.103(1.020-1.193)$ & 0.014 & $1.111(1.019-1.211)$ & 0.017 \\
\hline Hypertension (no) & $1.467(0.923-2.331)$ & 0.105 & $1.173(0.710-1.939)$ & 0.532 \\
\hline Dyslipidaemia (yes) & $1.100(0.949-1.275)$ & 0.206 & $1.141(0.969-1.344)$ & 0.113 \\
\hline Dyslipidaemia (no) & $1.105(1.009-1.210)$ & 0.031 & $1.116(1.011-1.233)$ & 0.030 \\
\hline Atrial fibrillation (yes) & $1.141(0.900-1.446)$ & 0.276 & $1.182(0.902-1.548)$ & 0.226 \\
\hline Atrial fibrillation (no) & $1.099(1.012-1.193)$ & 0.024 & $1.111(1.016-1.216)$ & 0.022 \\
\hline Stroke (yes) & $1.085(0.954-1.234)$ & 0.212 & 1.089 (0.942-1.259) & 0.248 \\
\hline Stroke (no) & $1.118(1.014-1.231)$ & 0.025 & $1.140(1.026-1.267)$ & 0.015 \\
\hline CKD (yes) & $1.021(0.869-1.199)$ & 0.805 & $0.998(0.834-1.194)$ & 0.982 \\
\hline CKD (no) & $1.131(1.036-1.235)$ & 0.006 & $1.153(1.046-1.270)$ & 0.004 \\
\hline \multicolumn{5}{|l|}{ Prescribed drugs } \\
\hline ACEI (yes) & $1.199(1.032-1.393)$ & 0.018 & $1.198(1.017-1.412)$ & 0.030 \\
\hline ACEI (no) & $1.068(0.976-1.169)$ & 0.151 & $1.087(0.984-1.200)$ & 0.099 \\
\hline BB (yes) & $1.130(1.015-1.259)$ & 0.025 & $1.167(1.030-1.323)$ & 0.015 \\
\hline $\mathrm{BB}(\mathrm{no})$ & $1.080(0.965-1.207)$ & 0.179 & $1.080(0.961-1.214)$ & 0.194 \\
\hline CCB (yes) & $1.133(1.034-1.241)$ & 0.007 & $1.141(1.032-1.261)$ & 0.009 \\
\hline CCB (no) & $1.005(0.868-1.165)$ & 0.945 & $1.034(0.879-1.217)$ & 0.684 \\
\hline Statin (yes) & $1.114(0.904-1.373)$ & 0.313 & $1.140(0.904-1.438)$ & 0.2681 \\
\hline Statin (no) & $1.103(1.015-1.199)$ & 0.021 & $1.117(1.019-1.223)$ & 0.018 \\
\hline Antiplatelet drugs (yes) & $1.080(0.971-1.202)$ & 0.156 & $1.082(0.960-1.219)$ & 0.196 \\
\hline Antiplatelet drugs (no) & $1.132(1.012-1.267)$ & 0.029 & $1.163(1.030-1.312)$ & 0.015 \\
\hline \multicolumn{5}{|l|}{ Operation type } \\
\hline Open repair (yes) & $1.069(0.855-1.338)$ & 0.558 & $1.009(0.830-1.226)$ & 0.930 \\
\hline Endovascular repair (yes) & $0.911(0.705-1.179)$ & 0.479 & $1.029(0.793-1.334)$ & 0.832 \\
\hline
\end{tabular}

AAA, abdominal aortic aneurysm; ACEI, ACE inhibitor; BB, beta-blocker; CCB, calcium channel blocker; CKD, chronic kidney disease; COPD, chronic obstructive pulmonary disease; PS, propensity score; TAA, thoracic aortic aneurysm; TAAA, thoracoabdominal aneurysm.

males regardless of whether they had COPD. The most common comorbidities in AA patients with COPD were hypertension, CAD, stroke, dyslipidaemia and CHF. These results are similar to those reported by Flessenkaemper $e t a l,{ }^{21}$ who suggested that risk factors such as male gender and $\mathrm{CAD}$ could be used to increase the efficiency of screening for AAA. We suggest that other risk factors, such as hypertension, stroke, dyslipidaemia and CHF, might be useful in future screening for AA in patients with COPD.

A survey of 231 patients with COPD by Ando et $a t^{22}$ reported that only $27(11.7 \%)$ had AA and $20(8.7 \%)$ had AAA. This finding contrasts with the results of our nationwide study, in which 2089 (64\%) patients with AAA also 


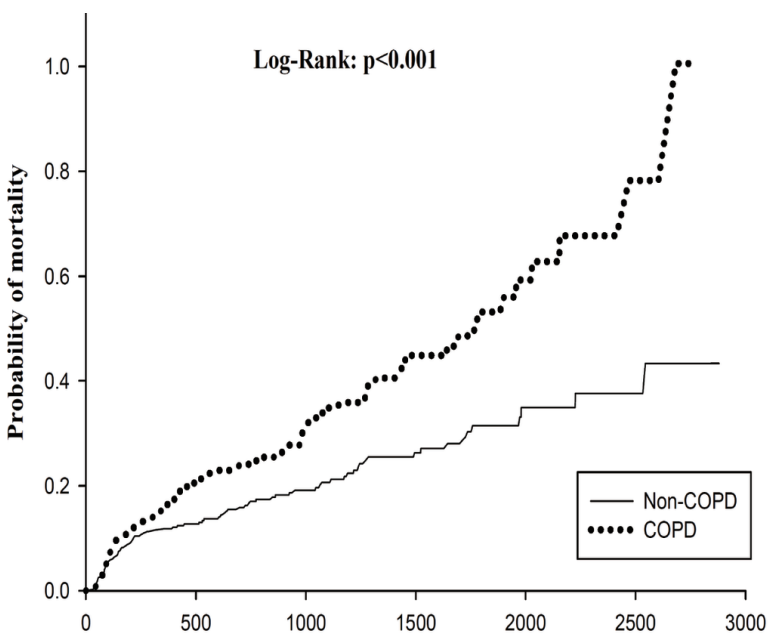

(A)

Follow-up (days)
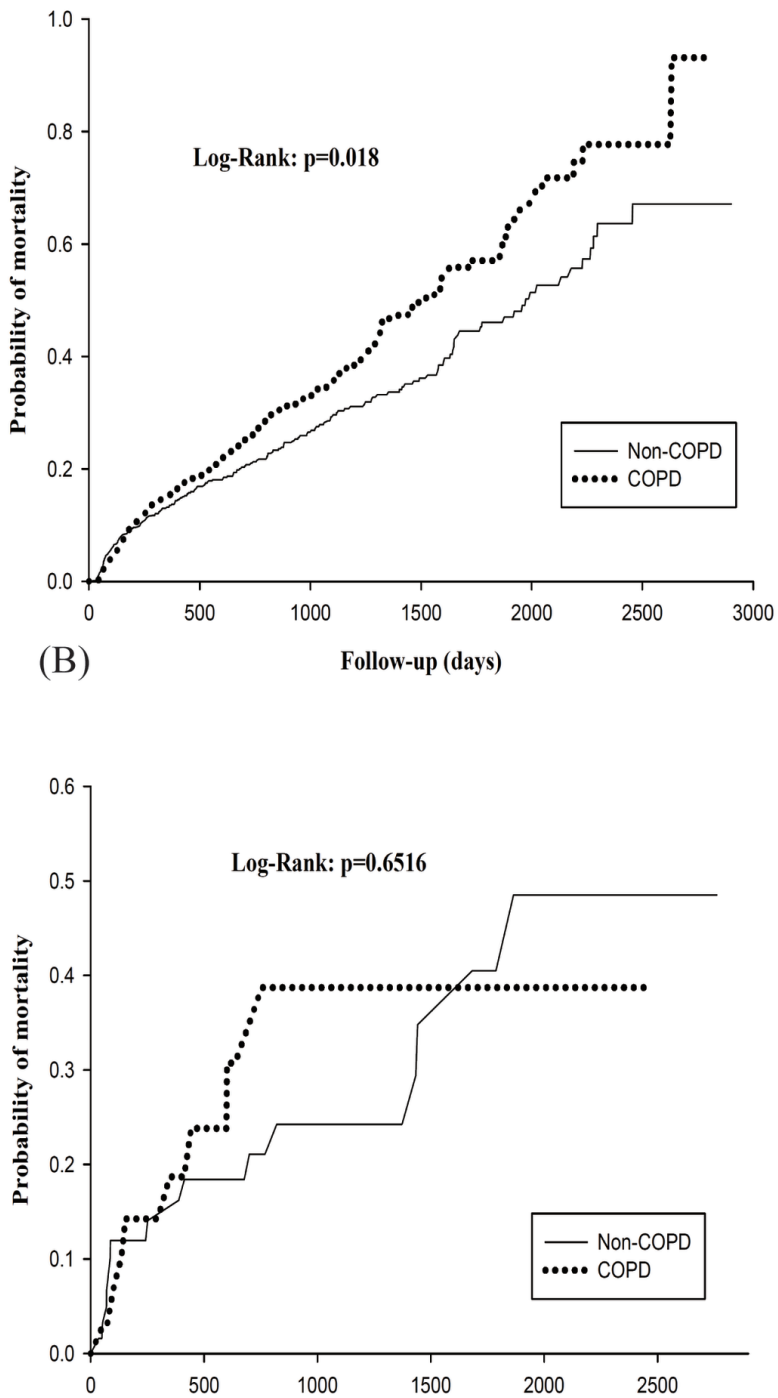

(C)
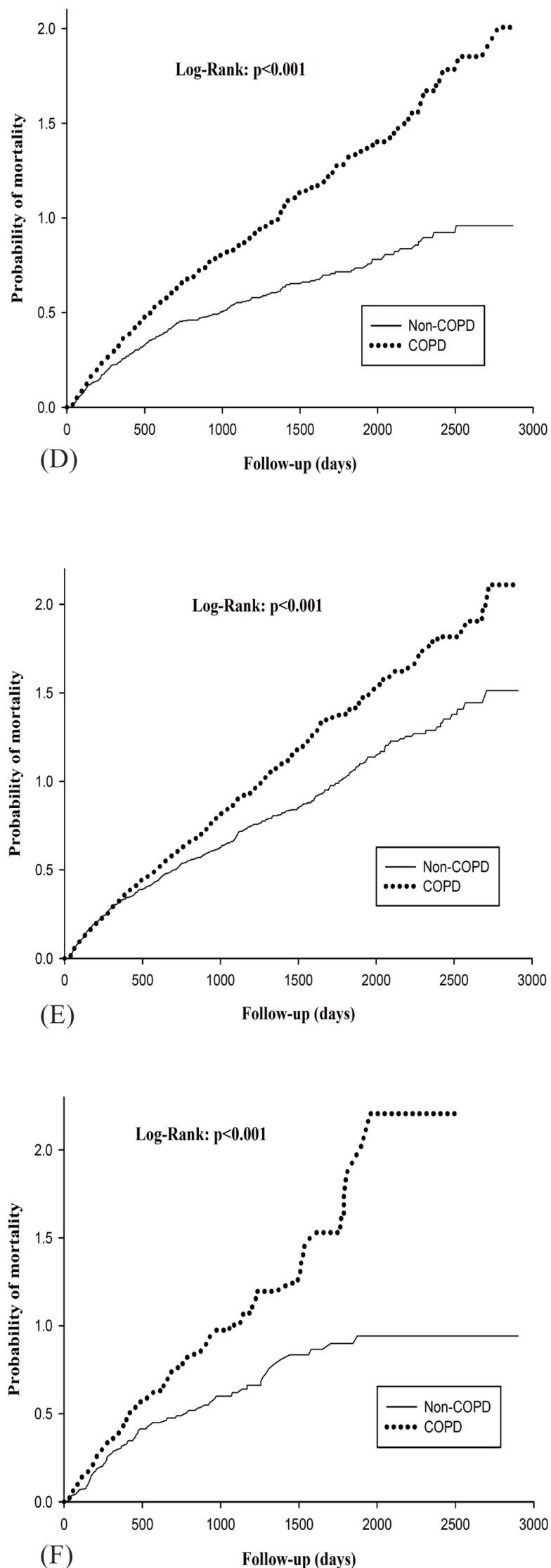

Figure 2 Cumulative risk of all-cause mortality between the patients with and without COPD. (A) Operation and TAA, (B) operation and AAA, (C) operation and TAAA, (D) non-operation and TAA, (E) non-operation and AAA and (F) non-operation and TAAA. AAA, abdominal aortic aneurysm; COPD, chronic obstructive pulmonary disease; TAA, thoracic aortic aneurysm; TAAA, thoracoabdominal aortic aneurysm. 
had comorbid COPD. To the best of our knowledge, with the exception of two studies with conflicting results, ${ }^{23} 24$ there have been no major reports on the prevalence or incidence of AA in the Asian population. Poon et $a l^{25}$ reported that the prevalence of AAA in Chinese patients was low and that their results did not support routine screening for AAA; however, another study showed that AAA was not uncommon and had a comparable incidence with that in the West. ${ }^{24}$ To clarify these issues, a study that includes more Asian countries and a larger population is needed.

\section{Prescription patterns}

Medical management is important in the control of AAs, with the main goal of therapy being to decrease shear stress by reducing blood pressure and contractility. ${ }^{26}$ In a small retrospective study, BBs were shown to effectively decrease AAA growth. ${ }^{27}$ In another study, prophylactic BBs were found to be associated with a slowing in the rate of aortic dilatation. ${ }^{28}$ Indeed, BBs can reduce left ventricular contractility and reduce shear stress in the aorta. However, a prospective randomised double-blind study showed that patients with AAA do not tolerate BBs and that these medications have no significant effect on the growth rates of small AAAs. ${ }^{29}$

In our study, before PSM, the most common medications for AA were CCBs, followed by diuretics and BBs. Although the prescribing rates of BBs were associated with significant differences between the patients with and without COPD, this difference did not persist after PSM. BBs are often prescribed for AA, but physicians may be concerned about contraindications and may fear inducing adverse reactions or bronchospasm, particularly in patients with obstructive airway disease. In our analysis, BBs were well tolerated in patients with AA and COPD and had a clear protective effect on reducing mortality. This is consistent with their use being recommended. Notably, the safety of BBs has long been proven in patients with $\mathrm{COPD}$, and there is a growing body of evidence from clinical trials showing that BBs should not be withheld in this patient group. ${ }^{30}$

\section{ACEl and ARB in AA}

In our analysis, ARBs, BBs, CCBs and diuretics, but not ACEIs, were associated with reduced mortality in patients with AA and COPD. In an animal model, AAs were associated with increased transforming growth factor- $\beta$ signalling, and the ARB losartan has been shown to block transforming growth factor- $\beta{ }^{31}$ Losartan can therefore prevent elastic fibre fragmentation and blunt transforming growth factor- $\beta$ signalling in the aorta, thereby reducing the growth rates of AAs. ${ }^{32}$ However, ACEIs were shown to prevent aortic dissection and the apoptosis of vascular smooth muscle cells in another animal model ${ }^{33}$; Hackam et al reported that ACEIs were protective against aortic expansion and rupture, whereas ARBs did not protect against AAA rupture. ${ }^{34}$ Other experimental evidence shows that ACEIs increase collagen synthesis, improve plaque stabilisation and diminish aortic stiffness. ${ }^{35}$ To further complicate matters, in a prospective cohort study of 1701 patients in the UK, Sweeting et $a l^{36}$ showed that aneurysm growth was faster in patients receiving ACEIs. This finding conflicts with previous research and observational data from Canada showing that ACEIs have protective benefits. ${ }^{35}$

Considering all the data, the inconsistent results regarding the efficacies of ARBs and ACEIs in reducing AA growth limit any meaningful conclusion. Undoubtedly, these problems result from differences in the models used, selection bias, unaccounted confounding factors and the multiple possible pathways of AA development.

A recently systematic review of the current data on pharmaceutical therapies for AAA showed that pharmaceutical therapies cannot halt AAA growth. ${ }^{37}$ Small AAA growth rates were lower than anticipated, and ACEI had no significant impact in reducing the small AAA growth rate. $^{38}$

\section{Limitations}

This study has some important limitations. Our study relies on diagnosed COPD; however, according to a previous study, ${ }^{1}$ a large proportion of the cases might be missed. We did not have access to data on vital signs (ie, blood pressure and heart rate) or to imaging results (ie, we could not estimate the size or progression of AAs). We also did not include data on pulmonary function tests or the severity of COPD, and we were unable to find a clear relationship between the size of AA and the severity of COPD. However, we focused on all-cause mortality, rehospitalisation rates and reoperation rates and performed a subgroup analysis (operation vs non-operation) to reduce bias. This was also a large nationwide study of all registered patients with AA in Taiwan, which should allow generalisation to other COPD populations. Finally, we also performed PSM, which reduced the bias in estimating the treatment effects and reduced the likelihood of confounding data. We excluded patients who died within 30 days and individuals with a COPD diagnosis after the index date. Additionally, chronic conditions such as COPD and aneurysms might have been present at the time of inclusion.

\section{CONCLUSIONS}

Improvements in the preoperative and postoperative management of patients with COPD undergoing major operation have resulted in reduced mortality and morbidity rates. However, although we showed improvement in the safety and outcomes of patients with COPD undergoing AAA repair, we also showed that the overall mortality remains higher than that in patients without COPD. In addition, we also observed high mortality rates among patients with COPD who did not undergo operation. Further research is clearly needed to identify the most appropriate therapy for reducing mortality in patients with AA and COPD. 
Contributors Substantial contributions to the conception or design of the work: $\mathrm{K}-\mathrm{ML}$ and $\mathrm{C}-\mathrm{YC}$. Acquisition, analysis and interpretation of data for the work: C-YC. Drafting the work and revising it critically for important intellectual content: K-ML and C-YC. Final approval of the version to be published: $\mathrm{K}-\mathrm{ML}$ and $\mathrm{C}-\mathrm{YC}$.

Funding This work was supported by the National Science Council (grant number 103-2320-B-037-014), Kaohsiung Medical University Hospital (KMUH103-3R69) and Chi Mei Medical Center, Chiali (CCFHR10503).

Competing interests None declared.

Ethics approval This study was approved by the Institutional Review Board of Kaohsiung Medical University Hospital (KMUH-IRB-EXEMPT-20130199).

Provenance and peer review Not commissioned; externally peer reviewed.

Data sharing statement № additional data are available.

Open Access This is an Open Access article distributed in accordance with the Creative Commons Attribution Non Commercial (CC BY-NC 4.0) license, which permits others to distribute, remix, adapt, build upon this work non-commercially, and license their derivative works on different terms, provided the original work is properly cited and the use is non-commercial. See: http://creativecommons.org/ licenses/by-nc/4.0/

(c) Article author(s) (or their employer(s) unless otherwise stated in the text of the article) 2017. All rights reserved. No commercial use is permitted unless otherwise expressly granted.

\section{REFERENCES}

1. Meijer CA, Kokje VB, van Tongeren RB, et al. An association between chronic obstructive pulmonary disease and abdominal aortic aneurysm beyond smoking: results from a case-control study. Eur $J$ Vasc Endovasc Surg 2012;44:153-7.

2. Darwood R, Earnshaw JJ, Turton G, et al. Twenty-year review of abdominal aortic aneurysm screening in men in the county of Gloucestershire, United Kingdom. J Vasc Surg 2012;56:8-13.

3. Ashton HA, Buxton MJ, Day NE, et al. The Multicentre Aneurysm Screening Study (MASS) into the effect of abdominal aortic aneurysm screening on mortality in men: a randomised controlled trial. Lancet 2002;360:1531-9.

4. Vainberg M. Screening for abdominal aortic aneurysm. Can Fam Physician 2012;58:253.

5. Axelrod DA, Henke PK, Wakefield TW, et al. Impact of chronic obstructive pulmonary disease on elective and emergency abdominal aortic aneurysm repair. J Vasc Surg 2001;33:72-6.

6. Jonker FH, Schlösser FJ, Dewan M, et al. Patients with abdominal aortic aneurysm and chronic obstructive pulmonary disease have improved outcomes with endovascular aneurysm repair compared with open repair. Vascular 2009;17:316-24.

7. Lvovsky D, Fulambarker A, Cohen ME, et al. Independent contributions of chronic obstructive pulmonary disease and abdominal aortic aneurysm to mortality risk. Chest 2005;128:265S.

8. Eskandari MK, Rhee RY, Steed DL, et al. Oxygen-dependent chronic obstructive pulmonary disease does not prohibit aortic aneurysm repair. Am J Surg 1999;178:125-8.

9. Wang SW, Huang YB, Huang JW, et al. Epidemiology, Clinical Features, and Prescribing Patterns of Aortic Aneurysm in Asian Population From 2005 to 2011. Medicine 2015;94:e1716.

10. NHIS working group. 2015 Taiwan National Health Interview and Medication Survey, Characteristics of completed sample (In Chinese). Taipei: Taiwan National Health Interview Survey Research Brief, 2015.

11. Cheng CL, Chien $\mathrm{HC}$, Lee $\mathrm{CH}$, et al. Validity of in-hospital mortality data among patients with acute myocardial infarction or stroke in National Health Insurance Research Database in Taiwan. Int J Cardiol 2015;201:96-101.

12. van Laarhoven CJ, Borstlap AC, van Berge Henegouwen DP, et al. Chronic obstructive pulmonary disease and abdominal aortic aneurysms. Eur J Vasc Surg 1993;7:386-90.

13. Lindholt JS, Heickendorff L, Antonsen S, et al. Natural history of abdominal aortic aneurysm with and without coexisting chronic obstructive pulmonary disease. J Vasc Surg 1998;28:226-33.

14. Cronenwett JL, Murphy TF, Zelenock GB, et al. Actuarial analysis of variables associated with rupture of small abdominal aortic aneurysms. Surgery 1985;98:472-83.
15. Johnston KW, Scobie TK. Multicenter prospective study of nonruptured abdominal aortic aneurysms. I. Population and operative management. J Vasc Surg 1988;7:69-81.

16. Compton CN, Dillavou ED, Sheehan MK, et al. Is abdominal aortic aneurysm repair appropriate in oxygen-dependent chronic obstructive pulmonary disease patients? J Vasc Surg 2005;42:650-3.

17. Lederle FA, Freischlag JA, Kyriakides TC, et al. Long-term comparison of endovascular and open repair of abdominal aortic aneurysm. N Engl J Med 2012;367:1988-97.

18. Lee K, Tang E, Dubois L, et al. Durability and survival are similar after elective endovascular and open repair of abdominal aortic aneurysms in younger patients. J Vasc Surg 2015;61:636-41.

19. Lederle FA, Freischlag JA, Kyriakides TC, et al. Open Versus Endovascular Repair (OVER) Veterans Affairs Cooperative Study Group. JAMA 2009;302:1535-42.

20. Qureshi MA, Greenberg RK, Mastracci TM, et al. Patients with chronic obstructive pulmonary disease have shorter survival but superior endovascular outcomes after endovascular aneurysm repair. $J$ Vasc Surg 2012;56:911-9.

21. Flessenkaemper IH, Loddenkemper R, Roll S, et al. Screening of COPD patients for abdominal aortic aneurysm. Int J Chron Obstruct Pulmon Dis 2015;10:1085-91.

22. Ando K, Kaneko N, Doi T, et al. Prevalence and risk factors of aortic aneurysm in patients with chronic obstructive pulmonary disease. $J$ Thorac Dis 2014;6:1388-95.

23. Poon JT, Cheng SW, Wong JS, et al. Prevalence of abdominal aortic aneurysm in Chinese patients with severe coronary artery disease. ANZ J Surg 2010;80:630-3.

24. Yii MK. Epidemiology of abdominal aortic aneurysm in an Asian population. ANZ J Surg 2003;73:393-5.

25. Poon JT, Cheng SW, Wong JS, et al. Prevalence of abdominal aortic aneurysm in Chinese patients with severe coronary artery disease. ANZ J Surg 2010;80:630-3.

26. Danyi P, Elefteriades JA, Jovin IS. Medical therapy of thoracic aortic aneurysms: are we there yet? Circulation 2011;124:1469-76.

27. Leach SD, Toole AL, Stern H, et al. Effect of beta-adrenergic blockade on the growth rate of abdominal aortic aneurysms. Arch Surg 1988;123:606-9.

28. Shores J, Berger KR, Murphy EA, et al. Progression of aortic dilatation and the benefit of long-term beta-adrenergic blockade in Marfan's syndrome. N Engl J Med 1994;330:1335-41.

29. Propanolol Aneurysm Trial Investigators. Propranolol for small abdominal aortic aneurysms: results of a randomized trial. J Vasc Surg 2002;35:72-9.

30. Albouaini K, Andron M, Alahmar A, et al. Beta-blockers use in patients with chronic obstructive pulmonary disease and concomitant cardiovascular conditions. Int J Chron Obstruct Pulmon Dis 2007;2:535-40.

31. Lavoie P, Robitaille G, Agharazii M, et al. Neutralization of transforming growth factor-beta attenuates hypertension and prevents renal injury in uremic rats. J Hypertens 2005;23:1895-903.

32. Habashi JP, Judge DP, Holm TM, et al. Losartan, an AT1 antagonist, prevents aortic aneurysm in a mouse model of Marfan syndrome. Science 2006;312:117-21.

33. Nagashima H, Uto K, Sakomura $Y$, et al. An angiotensin-converting enzyme inhibitor, not an angiotensin II type-1 receptor blocker, prevents beta-aminopropionitrile monofumarate-induced aortic dissection in rats. J Vasc Surg 2002;36:818-23.

34. Hackam DG, Thiruchelvam D, Redelmeier DA. Angiotensinconverting enzyme inhibitors and aortic rupture: a population-based case-control study. Lancet 2006;368:659-65.

35. Claridge MW, Hobbs SD, Quick CR, et al. ACE inhibitors increase type III collagen synthesis: a potential explanation for reduction in acute vascular events by ACE inhibitors. Eur J Vasc Endovasc Surg 2004;28:67-70.

36. Sweeting MJ, Thompson SG, Brown LC, et al. Use of angiotensin converting enzyme inhibitors is associated with increased growth rate of abdominal aortic aneurysms. J Vasc Surg 2010;52:1-4.

37. Kokje VB, Hamming JF, Lindeman JH. Editor's choice pharmaceutical management of small abdominal aortic aneurysms: a systematic review of the clinical evidence. Eur J Vasc Endovasc Surg 2015;50:702-13.

38. Bicknell CD, Kiru G, Falaschetti E, et al. An evaluation of the effect of an angiotensin-converting enzyme inhibitor on the growth rate of small abdominal aortic aneurysms: a randomized placebo-controlled trial (AARDVARK). Eur Heart J 2016;37:3213-21. 